\title{
The scenario of a single freak wave appearance in deep water - dispersive focusing mechanism framework
}

\author{
E. Pelinovsky ${ }^{1,2}$, E. Shurgalina ${ }^{1,2}$, and N. Chaikovskaya ${ }^{2}$ \\ ${ }^{1}$ Department of Nonlinear Geophysical Processes, Institute of Applied Physics, Nizhny Novgorod, Russia \\ ${ }^{2}$ Nizhny Novgorod State Technical University, Nizhny Novgorod, Russia
}

Received: 12 September 2010 - Revised: 6 November 2010 - Accepted: 16 November 2010 - Published: 11 January 2011

\begin{abstract}
One of the possible mechanisms of the emergence of freak waves in deep water, based on the dispersive focusing of unidirectional wave packets is analysed. This mechanism is associated with the frequency dispersion of water waves and manifested in the interference of many spectral components, moving with different velocities. Formation of a single freak wave in a random wind wave field is considered in the frame of linear theory. The characteristic lifetime of an abnormal wave in the framework of this mechanism for typical conditions is approximately two minutes, thus, a rapid effect is difficult to predict and prepare for. A rogue wave quickly changes its shape from a high ridge to a deep depression.
\end{abstract}

\section{Introduction}

The large-amplitude waves suddenly appearing for a short time on the sea surface (freak or rogue waves) attract the attention of professionals nowadays because of their danger to ships and oil platforms in sea, ports and tourist resorts on the coast. Numerous data of observing freak waves in different areas of the World Ocean can be found, for example, in books (Lavrenov, 2003; Kurkin and Pelinovsky, 2004; Kharif et al., 2009) and papers (Kharif and Pelinovsky, 2003; Didenkulova et al., 2006; Liu, 2007). Among the mechanisms of their appearance in the open sea the following ones are marked (Kharif et al., 2009): (a) a superposition of a large number of individual spectral components, which move with different speeds and in different directions (the dispersive and geometrical focusing); (b) nonlinear mechanisms of modulation instability, in particular, the Benjamin-Feir instability; and (c) interaction of sea waves with currents and wind flow.

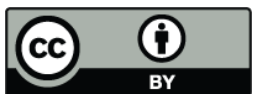

Correspondence to: E. Pelinovsky (pelinovsky@hydro.appl.sci-nnov.ru)
Each of these mechanisms has its own specificity, which is ultimately manifested in the probability of freak wave occurrence and the time of their life. It is possible that each mechanism leads to different forms of rogue waves and scenarios of their manifestation. All these important features have not been studied yet.

Here the possible scenario of the freak wave appearance in deep water, based on dispersive focusing of unidirectional wave packets is analysed. This mechanism is associated with the dispersion of water waves and is manifested in the interference of many spectral components, moving with different velocities. This mechanism "works" for both the deterministic (with certain conditions on the phases of spectral components) and random waves, leading to the appearance of abnormally high waves. It is possible in both, linear and nonlinear theories of water waves, although, of course, the nonlinearity leads to their peculiarities in the wave field (Pelinovsky and Kharif, 2000; Kharif et al., 2001; Pelinovsky et al., 2003; Shemer et al., 2007; Shemer and Dorfman, 2008). We also emphasize that the mechanism of dispersion focusing is very popular with experimentalists, because it allows generating a wave of huge height in a relatively short tank. The main attention in the laboratory experiments is paid to the description of the wave field (the displacement of water surface and particle velocities) at the focal point, which is essential for the subsequent assessment of the impact of extreme waves on ships and platforms (Brown and Jensen, 2001; Contento et al., 2001; Johannesen and Swan, 2001; Clauss, 2002; Touboul, 2006; Shemer et al., 2007; Shemer and Dorfman, 2008; Kharif et al., 2008, 2009; Shemer and Sergeeva, 2009).

Theoretical results for focusing wave packets in deep water are obtained mostly in the linear theory, in the framework of the so-called parabolic equation for the envelope of the wave packet (see, for example, Clauss and Bergmann, 1986; Magnusson et al., 1999; Pelinovsky and Kharif, 2000; Shemer et al., 2002; Pelinovsky et al., 2003; Shemer and

Published by Copernicus Publications on behalf of the European Geosciences Union. 

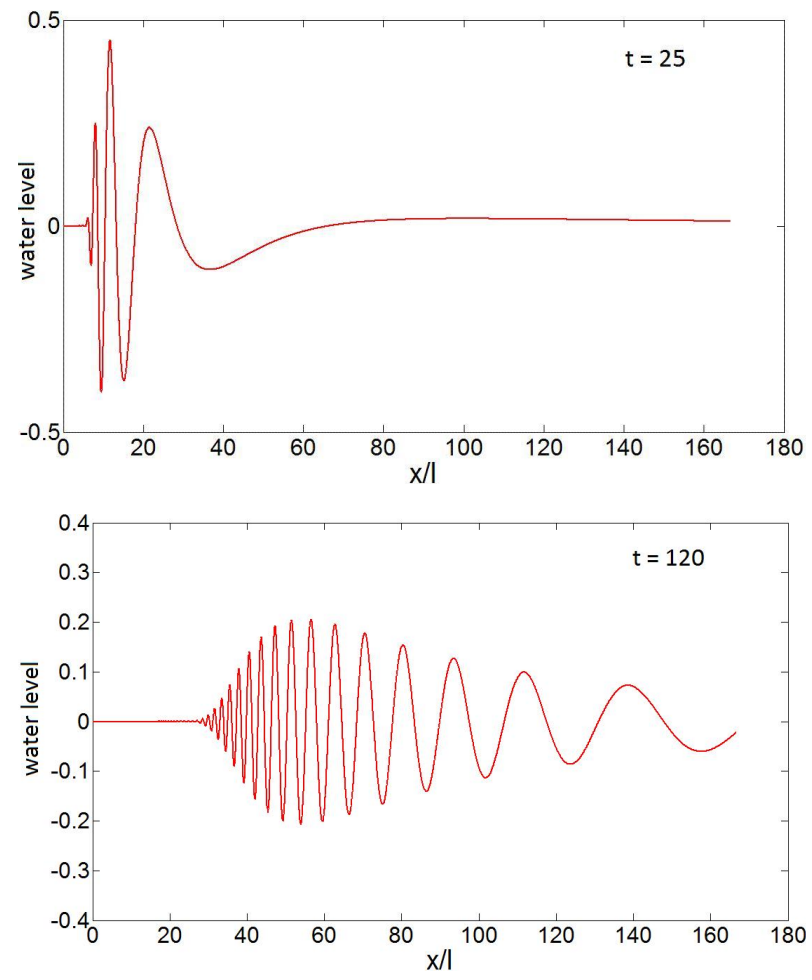

Fig. 1. Evolution of the Gaussian pulse in deep water for long times.

Dorfman, 2008). The particular analytical solution of this equation is the Gaussian packet, which demonstrates the process of the emergence of abnormally high waves and their disappearance. It is important to emphasize that the parabolic equation is valid for slow varying envelope on the scale of the carrier waves, so that the freak wave is a group of waves such as "Three Sisters" - a term often encountered in the witness descriptions of the phenomenon. However, it does not meet a single rogue wave, the description which is also present in the literature.

The aim of this work is to develop a scenario of appearance and disappearance of a single freak wave in the frame of the dispersive mechanism of focusing wave packets. Section 2 provides a solution to the Cauchy problem for waves in infinitely deep water, corresponding to the initial perturbation in the form of a single pulse. It is the basis for the demonstration of the occurrence of solitary freak waves in the deterministic wave field. The process of the appearing rogue waves in a random field of wind waves is considered in Sect. 3. It is shown that for typical conditions, the characteristic lifetime of a freak wave is about $2 \mathrm{~min}$, demonstrating the difficulties in predicting this dangerous phenomenon. Features of the script of the development of abnormal pulse are discussed in Sect. 4. It is shown that the freak wave is not only there for a short time, but quickly changes its shape from a high ridge to a deep depression. The results are summarized in Sect. 5.

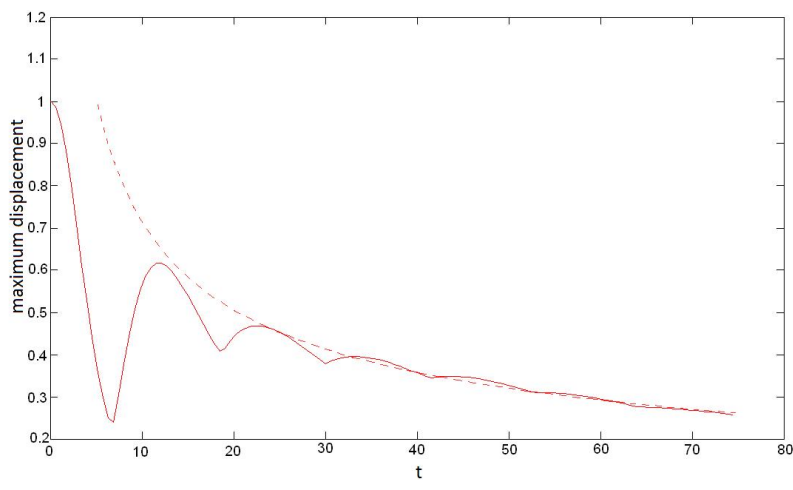

Fig. 2. The dependence of the maximum value of the wave field from dimensionless time (the solid line corresponds to the exact, and the dashed line - asymptotic solution).

\section{Generation of "huge" wave in a frequency-modulated wave packet}

The transformation of the wave packet into a single largeamplitude wave in the frame of the linear theory can be considered using the Fourier-superposition of spectral components. In practice, however, a different approach is used (Kharif et al., 2009): Cauchy problem is solved for the initial condition, which corresponding to the expected anomalous wave, and then the resulting solution is inverted in the space. As a result, possible forms of the wave packet can find the evolution of which leads to the formation of abnormal waves in a finite time, followed by its transformation back into the wave packet. Let us consider a classical solution of Cauchy problem for waves in deep water, written in the integral form

$\eta(x, \tau)=\int_{-\infty}^{+\infty} A(k) \exp \{i[\omega(k) \tau-k x]\} d k$,

where $\eta(x, \tau)$ is a displacement of the water level, $A(k)$ is Fourier spectrum determined by the initial disturbance, corresponding to the expected anomalous wave $\eta_{0}(x)$

$A(k)=\frac{1}{2 \pi} \int_{-\infty}^{+\infty} \eta_{0}(x) \exp (i k x) d x$,

$\omega(k)$ is a wave frequency determined from the dispersion relation of waves in deep water

$\omega(k)=\sqrt{g k}$,

where $g$ is gravity acceleration. Integral Eq. (1) analytically is not calculated for "reasonable" initial disturbances, but at long times, its presented by a well-known expression obtained by the method of stationary phase (Whitham, 1977)

$\eta(x, \tau) \approx Z(x, \tau) \cos \left[\omega(x, \tau) \tau-k(x, \tau) x+\varphi[k(x, \tau)]-\frac{\pi}{4}\right]$, 

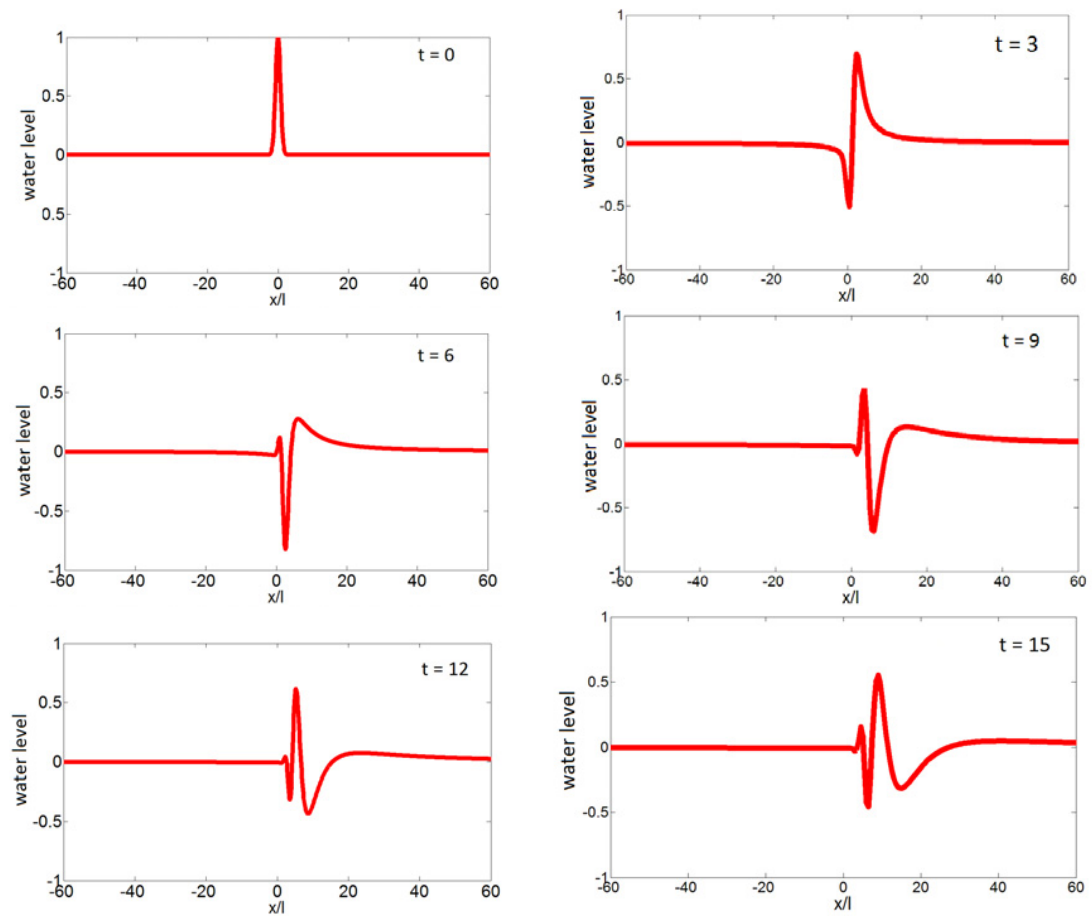

Fig. 3. Evolution of the pulse at short times.

$Z(x, \tau)=2 \sqrt{\frac{2 \pi}{\left|d C_{g r} / d k\right|}} \frac{|A(k)|}{\sqrt{\tau}}$,

$C_{g r}(k)=\frac{d \omega}{d k}=\frac{1}{2} \sqrt{\frac{g}{k}}=\frac{x}{\tau}$,

$|A|$ and $\varphi$ are module and an argument of the complex spectrum of $A(k)$. The last expression in Eq. (5) allows unambiguously to find the wave number $k(x, \tau)=g \tau^{2} / 4 x^{2}$, then from Eq. (3) the wave frequency $\omega(x, \tau)=g \tau / 2 x$. The final asymptotic expression for the wave field takes the following form

$\eta(x, \tau) \approx 2 \sqrt{\pi}\left|A\left(g \tau^{2} / 4 x^{2}\right)\right| \sqrt{\frac{g \tau^{2}}{x^{3}}} \cos \left[\frac{g \tau^{2}}{4 x}+\varphi-\frac{\pi}{4}\right]$

it describes, at each moment in time, the wave packet with variable amplitude and length (frequency-modulated wave train), and ahead follow longer wavelengths, which have a great group velocity. Asymptotic solutions for waves of any physical nature are well-known (Whitham, 1977) and, therefore, the details of their derivation are not discussed here.

As an expected anomalous wave it is natural to choose a Gaussian pulse with a characteristic amplitude $A_{0}$ and half of a length $l$

$\eta_{0}(x)=A_{0} e^{-\frac{x^{2}}{l^{2}}}$

Then at large distances $(x>>l)$ it transforms into a wave packet

$\eta(x, \tau) \approx A_{0} \frac{l \tau}{x} \sqrt{\frac{g}{x}} \exp \left(-\frac{g^{2} l^{2} \tau^{4}}{64 x^{4}}\right) \cos \left[\frac{g \tau^{2}}{4 x}-\frac{\pi}{4}\right]$.

The shape of the wave packet at different moments of the dimensionless time $(t=\tau \sqrt{g / l})$ is shown in Fig. 1. Over time a train stretches in the space (proportional to $\tau$ ), and its amplitude decreases as $\tau^{-1 / 2}$, ensuring the conservation of wave energy. The number of individual waves increases linearly with time, the wave of maximum amplitude retaining its length and speed of propagation.

At short times the integral Eq. (1) is calculated numerically, which allowed us to define the limits of applicability of the asymptotic solutions. As it turned out, at values of dimensionless time $\sim 20-25$, the maximum water displacement (the amplitude of the high ridge) is well described by the asymptotic value (Fig. 2).

It is clearly seen that in the frame of the exact solution, the maximum of the field decreases sharply at times of $\sim 5-10$ and, consequently, the wave in the form of the hump disappears for a while. The evolution of the wave shape at short dimensionless times is shown in Fig. 3.

Initially, a positive bell-shaped pulse is transformed into a wave of depression and further into the wave train. The quick change of polarity of the pulse had not previously been noted in the literature, however, as we show below, it plays an important role in the scenarios of freak wave formation. 

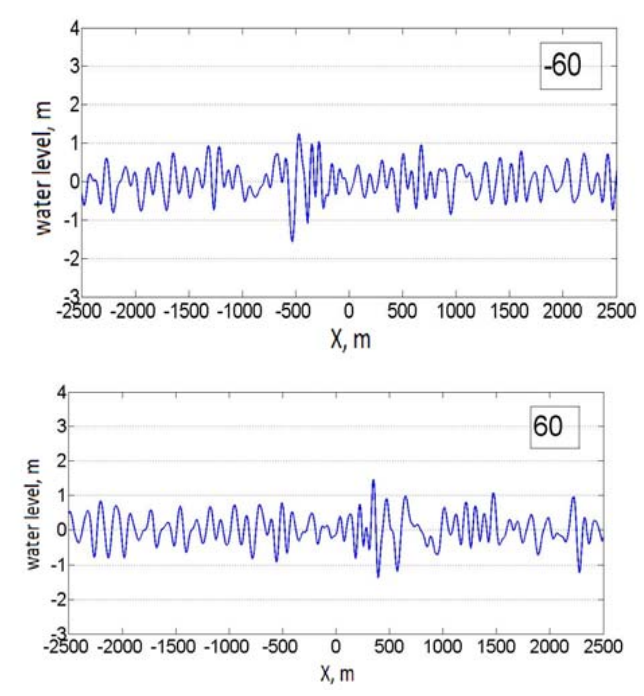

Fig. 4. Snapshots of the wave field at different times (s).

The solution given above describes the transformation of a solitary wave in the frequency modulated wave packet. If the wave packet is inverted in the space, so that now the short waves with small group velocity are ahead of the long ones, the wave packet will be transformed into a solitary wave of Gaussian shape. The property of inverting the solutions of linear equations of ideal hydrodynamics is used to find optimal conditions for the dispersive focusing. Nonlinearity, of course, affects the process of focusing. In particular, in the papers of Shemer et al. $(2006,2007)$ it was demonstrated that in an unidirectional focusing process nonlinear effects are essential in two important aspects. They may lead to a considerable modification of the complex amplitude spectrum in the course of evolution, affecting both absolute values of the amplitudes of various harmonics and their phases. The other aspect is related to the contribution of bound waves that changes considerably the amplitudes of troughs and crests and violates the symmetry between the two. But if the wave amplitude is relatively weak, this effect is not fundamental; it is just needed to make a few adjustments to the form of the wave packet (Johannesen and Swan, 2001; Clauss, 2002).

Concluding this section, we note that in laboratory conditions a single wave with a broad spectrum is generated by a wave maker with variable frequency, changing in finite limits according to the linear law (the optimal law for the generation of solitary waves through the mechanism discussed above) (see, for example, Brown and Jensen, 2001; Shemer et al., 2007; Shemer and Dorfman, 2008; Kharif et al., 2008). In this case, the signal spectrum is almost rectangular, while the wave itself (through the inverse Fourier transform) - crest of small, oscillating tails (like $\sin (x) / x$ ); it is the shape of a focused wave observed in experiments (Kharif et al., 2008; Shemer and Dorfman, 2008).

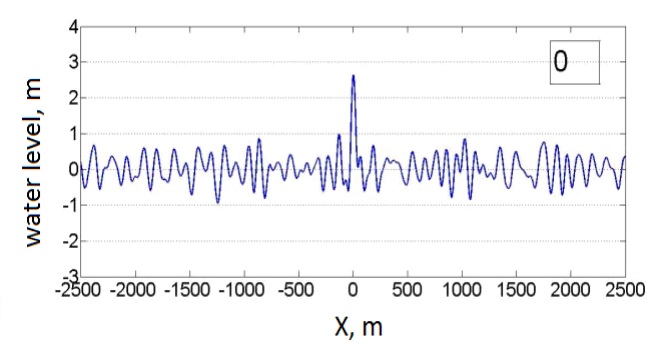

\section{Generation of a single pulse in a random field of wind waves}

The mechanism of dispersion focusing described above must occur in a random field of wind waves, the spectral components of which move with different velocities. A simple statistical analysis of a random superposition of waves with a narrow spectrum in the linear approximation leads to the Rayleigh distribution, so that the freak wave should appear once every $10 \mathrm{~h}$ (Dysthe et al., 2008; Kharif et al., 2009). The simulation of the wave field for such long times is rather a difficult task, so we assume that, along with random components, there is a deterministic frequency-modulated packet of small amplitude, as described above. Then by the linearity the random and regular components of wind wave field do not interact with each other, so that the process of forming a single pulse from a frequency-modulated packet follows the scenario described above. The random disturbance, on average, does not change its energy and the possibility of a big wave in it is small at relatively short times. As a result, the initial wave field "looks" purely random, and then there comes a high ridge, which over time is again "dissolved" in random waves. Such processes of interference of random and deterministic fields have already been discussed in the literature (Kharif et al., 2009), but not for the formation of a single wave on deep water. Nonlinearity, if it is weak, can not prevent the dispersive focusing of a deterministic wave packet, so it can be ignored on the first stage.

In numerical experiments the random wave field is set by the superposition of spectral components with random phases

$\eta(x, \tau)=\sum_{i=1}^{N} A_{i} \cos \left(\omega_{i} \tau-k_{i} x+\varphi_{i}\right)$, 

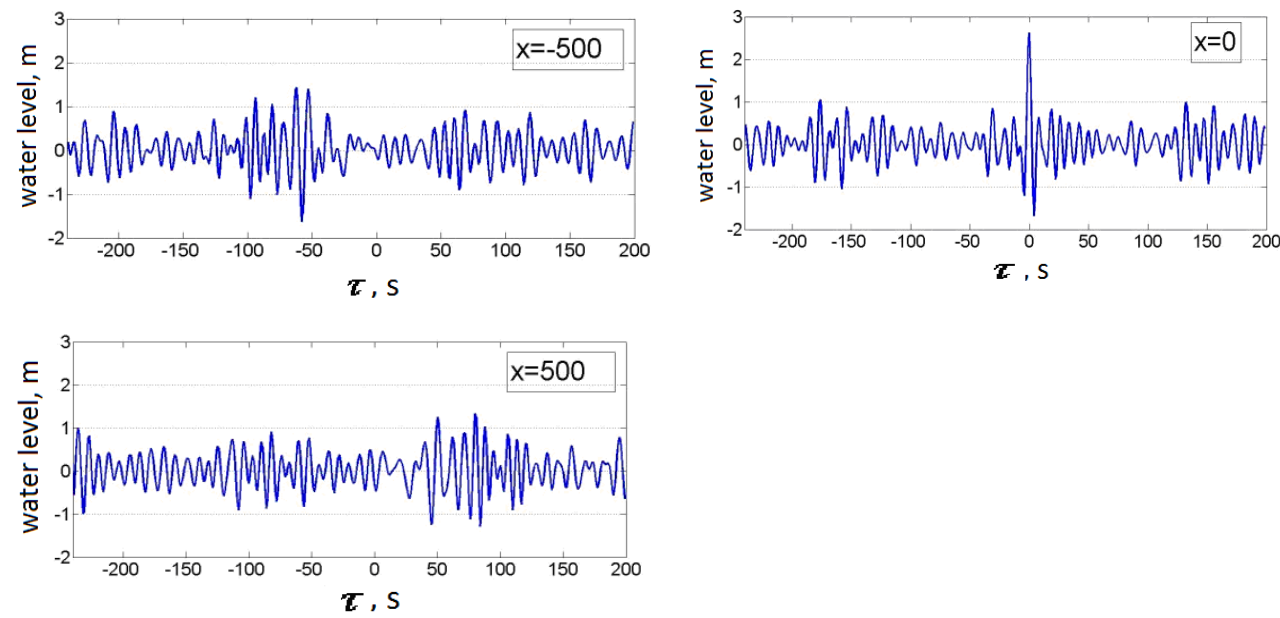

Fig. 5. Time series of the wave field at different distances (m) from the zone of anomalous wave.
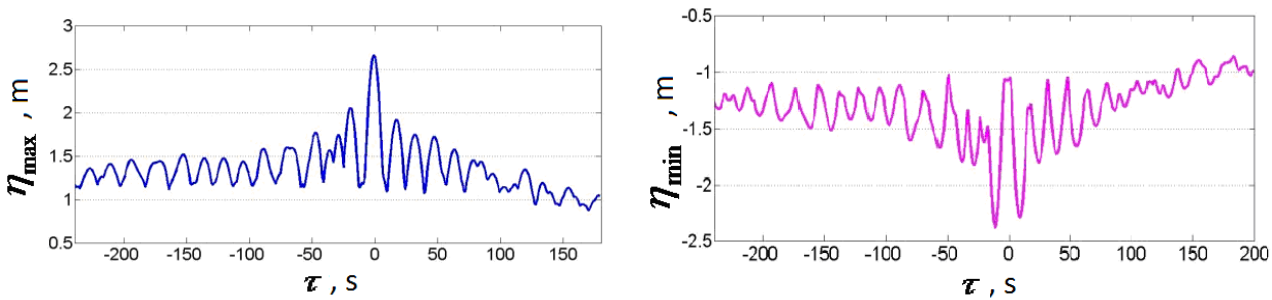

Fig. 6. Maximum values of positive and negative amplitudes versus time.

where the spectral amplitude $A_{i}=\sqrt{S(k) \Delta k}, \Delta k$ is a sampling interval of the spectrum, $k_{i}=i \Delta k, \omega_{i}=\sqrt{g k_{i}}, N=$ 156 is the total number of harmonics. Phases $\varphi_{i}$ are evenly distributed and set with a random number generator, $S(k)$ is energetic spectrum, for example, the spectrum of the Pearson-Moskowitz or JONSWAP spectrum. In our calculations, we used a simpler Gaussian approximation of the wind wave spectrum

$S(k)=B \exp \left(-\frac{\left(k-k_{0}\right)^{2}}{l^{2}}\right)$,

with $B=0.05 \mathrm{~m}^{3}, l=0.5 \mathrm{~m}^{-1}, k_{0}=0.063 \mathrm{~m}^{-1}$. In this case, the characteristic wavelength is $100 \mathrm{~m}$ and a significant height of the wave $H_{s}=1.5 \mathrm{~m}$, thus, the rms value of the surface elevation variation as $\sigma=H_{s} / 4$ yields to steepness $k_{0} \sigma \approx 0.025$. On the other hand the maximum height of the freak wave reaches $a_{\max }=2.7 \mathrm{~m}$ (see below) and, therefore, the steepness of the wave of maximum amplitude is $k_{0} a_{\max } \sim 0.17$. This nonlinearity may probably be sufficiently small to justify linear analysis if we compare the experimental results with Shemer et al. (2007) where the nonlinear effects are manifested at $k_{0} a_{\max } \sim 0.3$.

The superposition of deterministic and random components of the wave field at different times is illustrated in
Fig. 4, where the time (s) is measured from the onset of solitary wave of large amplitude. As we see, even one minute before, the abnormal waves are not visible and they also almost disappear in $1 \mathrm{~min}$, so the forecast of freak wave is indeed very difficult and, most importantly, there is little time to prepare for its appearance in such a short time. What is said above is also shown by the time series of the wave field at short distances $(500 \mathrm{~m})$ from the place of an abnormally large wave (Fig. 5).

A presentation of the typical lifetime of the rogue-wave is given in Fig. 6, which shows the maximum height of the ridges and deep depressions in the domain of $5 \mathrm{~km}$ versus time. As it can be seen, a significant change in the wave height occurs within about $2 \mathrm{~min}$, and this value can be taken for the lifetime of the anomalous wave. We emphasize that the freak wave appears both in the form of a high ridge and in form of a deep depression, and near the estimated time the wave changed its polarity several times.

\section{The scenario of a freak wave appearance}

The calculations given above show that the freak wave exists about $2 \mathrm{~min}$, and a detailed chronology during that time (the script of the process) will allow for the evolution of the freak 

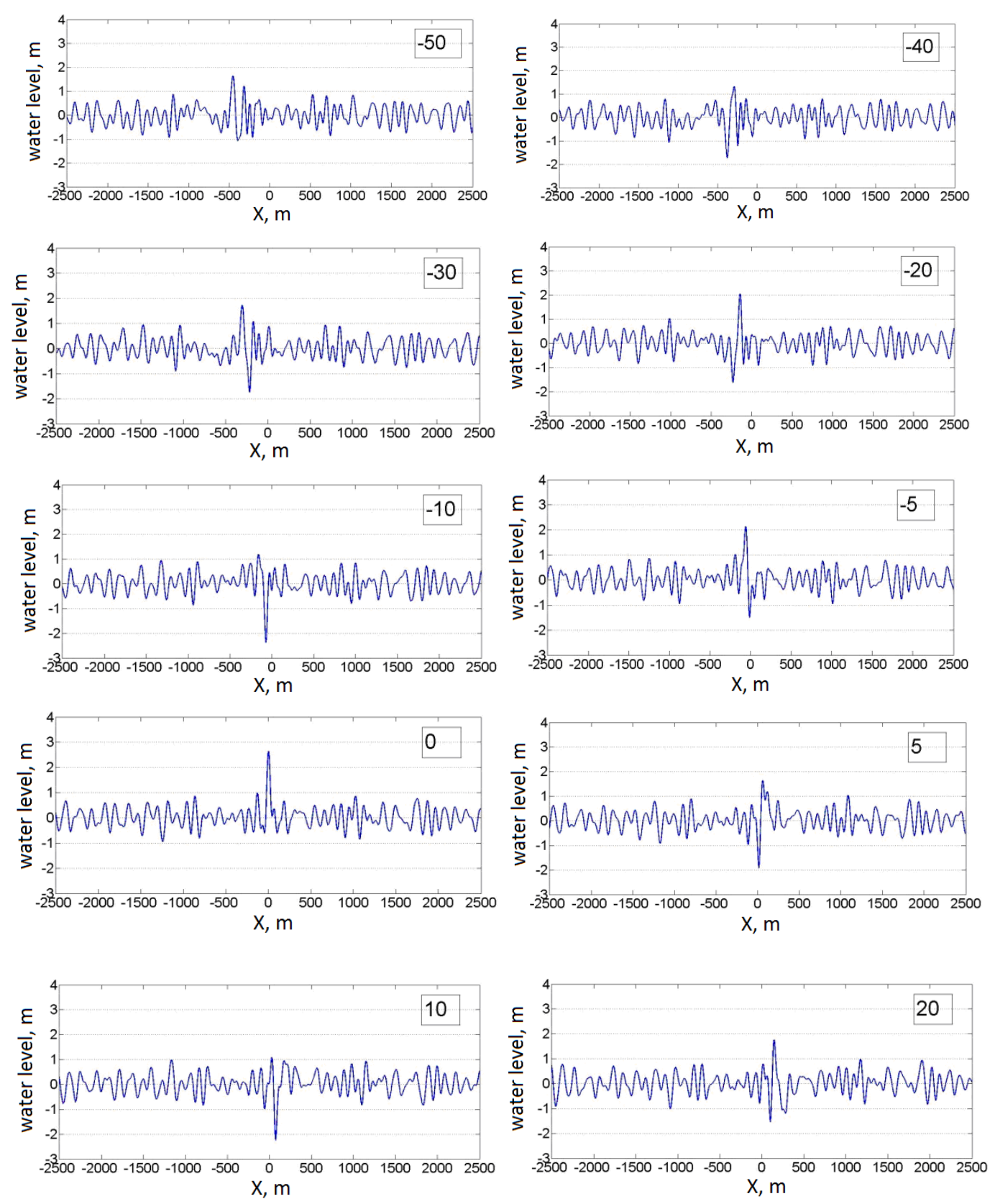

Fig. 7. Snapshots of water level for different times (s).

wave. Figure 7 shows the shape of the water surface at different times, calculated by the above formulas. At this time interval (about $2 \mathrm{~min}$ ) an anomalous solitary wave is always visible in the background of random wind waves. Its shape is constantly changing from crest to trough and back quite quickly (within approximately $10 \mathrm{~s}$ ).

Observability of the rogue waves depends on many factors. For example, if the observer is on the plane, he can only see one of the images shown in Fig. 7, and to estimate the height of the anomalous wave from its trough to the crest. In fact, such pictures of freak-waves have been received from space (Kharif et al., 2009) and are not discussed here. Freak waves were originally described in legends, transmitted orally from sailors who having survived the horror of meeting with this terrible phenomenon, hurried to share their impressions with others. Naturally, most of the stories contained a considerable exaggeration and fictitious descriptions. Having now a model of freak waves, it is possible to develop a "standardized" description schematizing the reaction of sailors to this phenomenon. 
Thus, in the case of the focusing wave packets, as shown above, the apparent abnormal wave in the form of a crest appears in approximately $1 \mathrm{~min}$ prior to its approach to the ship at the distance about 600-700 m. If the observer is on board, who also "prescribes" water level fluctuations in pitching, he can only see a big crest. This crest can be seen a few times (2-4 times) for about $10 \mathrm{~s}$, before it comes to the ship. In this case, the first not the highest ridges will hardly attract the attention of seafarers and, in fact, the freak waves will be visible for about $30 \mathrm{~s}$ before meeting with the biggest wave. And only when the wave appears just before the ship, the observer can see that the wave consists of a crest and trough or of trough/crest.

That is why phrases of the descriptions are typical: "crews do not have time to prepare for the meeting with the danger" (Kharif et al., 2009), which greatly aggravates the consequences of the meeting with the elements. The fact of the sudden appearance of the freak waves requires from the crew of any vessel not only professional knowledge, but also mental preparation. The reaction of seafarers, of course, depends on their experience related to stressful situations, such as during a storm. There are specific, purely psychological factors (the so-called sthenic or asthenic emotions, ability to anticipate situations in life and willingness to encounter them and so on). According to (Rogovin and Karpova, 1985), the willingness of action to external irritants is 0.5-2 s later, thus, there is no time to be prepared for a meeting with the freak wave. Therefore, one important task is to study the psychological characteristics of human behaviour in case of meeting with a freak wave and the development of special techniques and simulators for the crew of ships. In addition to purely technical issues (stability of the ship in large waves, a special lashing, etc.) this will prevent the severe consequences of this type of maritime disasters.

We should point out that in our study the temporal evolution problem is considered. The relation between the temporal and the spatial formulation was considered in detail in Shemer and Dorfman (2008). While the temporal approach is simpler and more "natural" for numerical simulations, it can not be realized in experiments where the evolution is spatial and the initial conditions are prescribed at $x=0$ rather than at $t=0$. It is possible that spatial formulation would be more appropriate from the point of view of the ship's captain watching the approaching waves, as attempted in the present manuscript. But in linear approximation, both approaches lead to the same results. The study by Shemer et al. (2010) indicated that for wider spectrum the importance of nonlinear effects seems to decrease and, therefore, we may use the temporal approach to analyse the scenario of appearance of the single freak wave.

Let us note that here we have considered the case when the wave of maximum amplitude is a ridge. Quite similarly, we can investigate a freak wave in the form of a deep depression. In the framework of linear theory, it is sufficient to change the sign in the Eq. (7). The scenario of the appearance of a freak wave in this case is almost not changed: waves of large amplitude are noticeable for about a minute and they change their polarity, appearing and disappearing at the sea surface for a short time. However, directly at the ship it will manifest itself in the form of a wave of deep depression and it will fail in trough. To predict the polarity of the freak wave (crest or trough), in the framework of this approach, is impossible if we use only the observation of waves in previous times.

\section{Conclusions}

The appearance of abnormally large waves on the sea surface is due to the different physical mechanisms. In this paper, we discuss the dispersive focusing scenario of a single freak wave formation. For typical conditions, it is shown that the characteristic lifetime of the freak waves is about two minutes. It is noted that at this time, the wave quickly (in about $10 \mathrm{~s}$ ) changes its shape from crest to trough and back. At the same time, for an observer onboard a ship, when only the high ridges are seen, the appearance of a freak wave is always unexpected, especially because about a minute before a large wave, it appears only 2-4 times, each time for $10 \mathrm{~s}$. The probability that the ship will rise to the top of the wave (if it is a crest), or fail in to the hole (if it has a negative polarity), is the same, and can not be determined in advance. All these points to the inherent difficulties in forecasting a freak wave, even a short time before when large waves become apparent on the sea surface.

Acknowledgements. The partial support of RFBR (08-05-00069), the European Programme FU-7 (No. 234175), State Contracts (No. 02.740.11.0732 and 01.420.1.2.0006) and RAS program "Nonlinear Dynamics" is used. Authors are also grateful to Lev Shemer (Tel-Aviv University, Israel) for the useful remarks.

Edited by: C. Kharif

Reviewed by: P. Liu and two other anonymous referees

\section{References}

Brown, M. G. and Jensen, A.: Experiments on focusing unidirectional water waves, J. Geophys. Research, 106, C8, 1691716928, 2001.

Clauss, G.: Dramas of the sea: episodic waves and their impact on offshore structures, Appl. Ocean Res., 24, 147-161, 2002.

Clauss, G. and Bergmann, J.: Gaussian wave packets: a new approach to seakeeping tests of ocean structures, Appl. Ocean Res., 8, 190-206, 1986.

Contento, G., Codigla, R., and D'Este, F.: Nonlinear effects in 2D transient non-breaking waves in a closed flume, Appl. Ocean Res., 23, 3-13, 2001.

Didenkulova, I. I., Slunyaev, A. V., Pelinovsky, E. N., and Kharif, C.: Freak waves in 2005, Nat. Hazards Earth Syst. Sci., 6, 10071015, doi:10.5194/nhess-6-1007-2006, 2006.

Dysthe, K., Krogstad, H. E., and Muller, P.: Oceanic rogue waves, Annu. Rev. Fluid Mech., 40, 287-310, 2008. 
Johannesen, T. B. and Swan, C.: A laboratory study of the focusing of transient and directionally spread surface water waves, Proc. Royal Soc. London, A457, 971-1006, 2001.

Kharif, C. and Pelinovsky, E.: Physical mechanisms of the rogue wave phenomenon, European J. Mechanics/B - Fluid, 22, 6, 603-634, 2003.

Kharif, C., Pelinovsky, E., and Slunyaev, A.: Rogue waves in the ocean, Springer, 196 pp., 2009.

Kharif, C., Pelinovsky, E., Talipova, T., and Slunyaev, A.: Focusing of nonlinear wave group in deep water, JETP Lett., 73, 4, 190195, 2001.

Kharif, C., Giovanangeli, J.-P., Touboul, J., Grare, L., and Pelinovsky, E.: Influence of wind on extreme wave events: Experimental and numerical approaches, J. Fluid Mech., 594, 209-247, 2008.

Kurkin, A. A. and Pelinovsky, E. N.: Freak waves: facts, theory and modelling, Nizhny Novgorod, Nizhny Novgorod Technical University Press, 157 pp., 2004.

Lavrenov, I. V.: Wind waves in ocean: dynamics and numerical simulations, Springer, Heidelberg, 377 pp., 2003.

Liu, P. C.: A chronology of freaque wave encounters, Geofizika, 24, 1, 57-70, 2007.

Magnusson, A. K., Donelan, M. A., and Drennan, W. M.: On estimating extremes in an evolving wave field, Coast. Eng., 36, 147-163, 1999.

Pelinovsky, E. and Kharif, C.: Simplified model of the freak wave formation from the random wave field, Proc. 15th Int. Workshop on Water Waves and Floating Bodies, Israel, 142-145, 2000.

Pelinovsky, E. N., Slunyaev, A. V., Talipova, T. G., and Kharif, C.: Nonlinear parabolic equation and extreme waves on the sea surface, Radiophys. Quantum. El., 46, 7, 451-463, 2003.
Rogovin, M. S. and Karpova, E. V.: Content dynamics and tier organization of concepts in the psychological analysis of subjective time, Questions of Psychology, 3, 98-107, 1985 (in Russian).

Shemer, L., Goulitski, K., and Kit, K.: Steep transitional waves in tanks, Proc. $25^{\text {th }}$ OMAE, ASME paper OMAE2006-92547, Hamburg, 2006.

Shemer, L., Goulitski, K., and Kit, E.: Evolution of wide-spectrum wave groups in a tank: an experimental and numerical study, Eur J. Mech. B/Fluids, 26, 193-219, 2007.

Shemer, L. and Dorfman, B.: Experimental and numerical study of spatial and temporal evolution of nonlinear wave groups, Nonlin. Processes Geophys., 15, 931-942, doi:10.5194/npg-15-9312008, 2008.

Shemer, L., Kit, E., and Jiao, H.-Y.: An experimental and numerical study of the spatial evolution of unidirectional nonlinear waterwave groups, Phys. Fl., 14, 3380-3390, 2002.

Shemer, L. and Sergeeva, A.: An experimental study of spatial evolution of statistical parameters in a unidirectional narrowbanded random wavefield, J. Geophys. Res., 114, C01015, doi:10.1029/2008JC005077, 2009.

Shemer, L., Sergeeva, A., and Liberzon, D.: Effect of the initial spectrum on spatial evolution of the statistics of unidirectional nonlinear random waves, J. Geophys. Res., 115, C06326, doi:10.1029/2010JC006326, 2010.

Touboul, J., Giovanangeli, J. P., Kharif, Ch., and Pelinovsky, E.: Experiments and simulations of freak waves under the action of wind, Eur. J. Mech. B/Fluids, 25, 5, 662-676, 2006.

Whitham, G. B.: Linear and nonlinear waves, Wiley \& Sons, New York, 635 pp., 1974. 